\title{
Illusory contours and spatial judgment
}

\author{
NICOLE SCHOUMANS and ANNE C. SITTIG \\ Delft University of Technology, Delft, The Netherlands
}

\begin{abstract}
We investigated whether, in the human visual system, the mechanisms responsible for relative location judgments are the same when those judgments are made in the context of illusory contours and in the context of mentally joining two points. We asked subjects to align a dot with the oblique contour of an illusory surface or to align a dot with two markers at an oblique orientation. The systematic errors differed in direction for these two conditions. All the systematic errors were orientation dependent. The errors in aligning a dot with an illusory contour seem to be related to the asymmetrical shape of the single objects, which are able to induce an illusory contour, as well as figure-ground segregation.
\end{abstract}

In everyday life, we make many judgments about intermediate positions - for example, arranging the items on our desk, estimating the situation when playing soccer, or recognizing a horse standing behind a fence or a caterpillar lying on a leaf. We can judge whether three objects are aligned or whether they define a plane. We can also estimate how a contour probably continues at those points at which the contour is masked by other objects or is indistinguishable from the background. All of these situations seem quite different. Nevertheless, in all cases the visual system can estimate the simplest connection between two objects. In this paper, we examine whether the mechanisms underlying judgment of that simplest connection are the same under different conditions.

As was already mentioned, the human visual system is capable of making estimates of intermediate positions. For example, when two objects are present in the visual field, we would not normally perceive a connecting line with an immediate visible presence. Nevertheless, we can judge in which direction one object is located with respect to the other and can thus reconstruct a straight line that could connect the two objects, and therefore, we can also judge whether or not a third object is aligned with two other objects.

In other cases, we can recognize contours while they are partially masked. Moreover, sometimes people can spontaneously perceive connecting contours where none exist. One example, a Kanizsa square (Kanizsa, 1955), is shown in Figure 1. A "whiter than white" surface with clear contours is perceived as an inner figure that could fit inside the gaps of the pacman-shaped objects. Such contours are called illusory contours. These contours are generally believed to be a result of mechanisms related to the

The authors thank Astrid Kappers and Jan Koenderink for their many useful critical remarks on this paper and Ruth Green for improving the language and clarity of the paper. Correspondence concerning this article should be addressed to N. Schoumans c/o A.M.L. Kappers, Princetonplein 5,3584 CC, Utrecht, The Netherlands (e-mail: nicoles@ hetnet.nl). recognition of (the shape of) objects, despite the presence of occluders (Grossberg, 1994; Heitger \& von der Heydt, 1993; Marr, 1982; Spillman \& Dresp, 1995). The definition of illusory contours that we shall follow is the one given by Petry and Meyer (1987, chap. 1). They state three defining characteristics for illusory contours. First, there is a sense of a bounded surface that has some property that differentiates it from an abutting or surrounding surface. Second, there is a sense of a boundary or edge around this surface. Third, the edge and surface connect or continue through discontinuities of the inducing pattern.

Illusory contours were first reported by Schumann (1900), and the occurrence of illusory contours has been studied by many researchers (for reviews, see Petry \& Meyer, 1987, and Spillman \& Dresp, 1995) because it is believed to be an important clue to how the human visual system works. It is not the aim of this study to provide a theory as to how the illusory contours arise but to examine how the estimate of the simplest connection between two objects is influenced by changing the context from that of mentally joining two points to one with an illusory contour. More specifically, we look at the similarities and differences in the biases of aligning a dot with an illusory contour and of aligning a dot to two other objects.

We made use of the fact that larger variable and systematic errors occur in tasks performed with an oblique orientation than in the same tasks performed with horizontal or vertical orientation. This effect is known as the oblique effect (see Appelle, 1972, for a review). Sittig and De Graaf (1994) reported results of a three-dot alignment task. They measured a strong orientation dependency in this task. With horizontal and vertical orientation, their subjects made no systematic errors but only variable errors in alignment. With an oblique orientation, the subjects systematically placed the middle dot below the straight line that could connect the two outer dots. If this alignment bias is independent of the context, we should find similar results when aligning a dot with an illusory contour.

On the other hand, there are many reports in the literature that show a great similarity in results in tasks with 

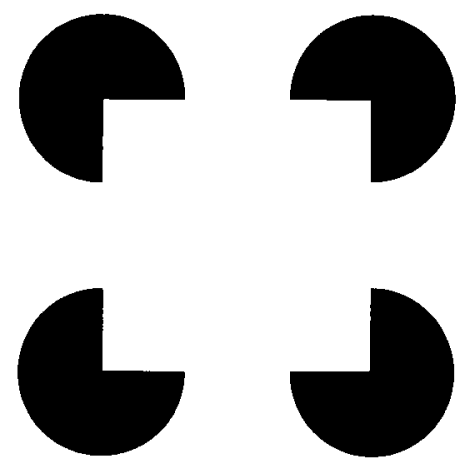

Figure 1. A Kanizsa square. The contours of a square are perceived even where no physical contours are present.

real and illusory contours (e.g., Gregory, 1972; Pomerantz, Goldberg, Golder, \& Tetewsky, 1981; Smith \& Over, 1977; Vogels \& Orban, 1987). These reports suggest that real and illusory lines are functionally equivalent at a certain level. This could indicate that aligning a dot with an illusory contour is similar to aligning a dot with a real contour, where the distance between the contour and the dot can be minimized. This should result in smaller variable errors, since aligning a dot with a real contour can be done very precisely.

The aim of this study is to examine whether the mechanisms responsible for relative location judgment are the same when those judgments are made in the context of illusory contours and in the context of mentally joining two points. In this paper, we describe the psychophysical experiments we did for this purpose. In our first experiment, we compared the results of aligning a dot with an oblique illusory contour and of aligning a dot with two other dots. Three more experiments were done to test hypothesized explanations of the first results.

\section{EXPERIMENT 1}

\section{Method}

Subject. Seven subjects, ( 4 female, 3 male), who all gave informed consent, participated in this experiment. Their ages varied from 22 to 28 years. Five subjects were naive as to the purpose of the experiment. Two subjects (N.S., R.B.) were aware of the purpose of the experiment, but their results did not differ from the results of the other subjects. All the subjects had normal or corrected-to-normal eyesight.

Task. Before the actual experiment, the experimenter explained the task and showed examples of the stimuli to the subjects. The experimenter also asked the subjects to report what they saw when they were shown four circular white discs with a gap of $90^{\circ}$ (four pacmen) on a black background, so grouped that a square surface could fit inside the gaps. All the subjects reported seeing a black square that was darker than the background.

The subjects had to align a white dot that appeared near the middle of two markers (specified in one of a number of ways). They could move the dot by pressing the arrow keys on a keyboard. The subjects' task was to align the dot as accurately as they could with an illusory contour, if present, or else to place the dot on what they thought would be the connecting line between the two outer markers. The dot could only be moved in a direction perpendicular to the line that had to be judged.

Apparatus. The stimuli were computer controlled and displayed on a screen with a pixel resolution of $0.27 \times 0.27 \mathrm{~mm}^{2}$. The screen had a black cardboard annulus in front of it, so that only the inner circular part of the screen was visible. This was done to exclude possible reference directions indicated by the edges of the screen. The screen was viewed from a distance of $60 \mathrm{~cm}$.

Stimuli. The stimuli that specified that with which the dot must be aligned in this experiment are sketched in Figures 2-4: a Kanizsa square placed on one of its corners (Kanizsa diamond, Figure 2, panel A), two dots determining an oblique straight line that could connect these dots (panels $2 \mathrm{~B}-2 \mathrm{C}$ ), two pacmen that specified one of the four contours of the diamond (half Kanizsa diamond, Figure 3), and an arrangement of four pacmen with two of them rotated by $90^{\circ}$ so that the four discs did not enclose a diamond (false Kanizsa diamond, Figure 4). All the stimuli indicated an oblique orientation at an angle of $45^{\circ}$ with the vertical. Each stimulus had a small dot, which could be repositioned by the subject near to the midway position between two outer objects. The distance between the outer objects subtended $9.5^{\circ}$ of visual angle, which corresponds to a distance of $10 \mathrm{~cm}$ on the computer screen. The stimuli were white and appeared on a dark background. The white pacmen had a radius of $2 \mathrm{~cm}$ ( $1.9^{\circ}$ of visual angle). The dots were single white pixels.

Procedure. The angle between the straight line connecting the starting position of the dot and the center of one of the outer markers and the straight line between the centers of the outer markers was chosen randomly and varied between $6^{\circ}$ in either direction (see Figure 5). The stimuli were presented in pseudo-random order. The whole set of stimuli was presented 10 (L.V., P.E., M.V.), 11 (Y.M., R.B., A.S.), or 12 (N.S.) times to the subjects.

The subjects were told to keep their heads upright at all times during the experiment. Eye movements were allowed. The experiment took place in a dark room so that only the stimuli were visible. The experiment lasted about half an hour.

Analysis. We recorded the angle between the straight line connecting the centers of the outer two markers and the line between the center of one of the outer markers and the adjusted position of the dot (see Figure 5). Note that this is an angle in the stimulus plane and not the visual angle. This angle was equal to zero when the adjustable dot was placed exactly on the specified line segment. The convention for signs is such that a positive value indicates that the dot was placed above the actual straight line that would connect the outer two markers.

\section{Results}

Figures 2, 3, and 4 summarize the results of all the subjects in all the conditions. To make it easier to see where the subjects put the adjustable dot when instructed to align it with an illusory contour or to align it with two markers, we present these results superimposed on a schematic outline of the respective stimuli. For example, Figure $2 \mathrm{~A}$ shows the results in the four cases in which a Kanizsa diamond was presented and the dot appeared near one of its four illusory contours, and Figure 2B shows the results in one case in which two dots plus the adjustable dot were presented. On each alignment line of the stimuli bars indicating the various subjects, systematic errors in degrees $\left({ }^{\circ}\right)$ were plotted. The direction of the bars indicates the true direction of the systematic errors. The whiskers indicate the standard error mean.

We averaged the mean results of the individual subjects. We also performed a two-tailed $t$ test, to test whether this value equaled zero. The results are shown in Table 1 .

Comparison of the Kanizsa-diamond task and the three-dot alignment task. Figure $2 \mathrm{~A}$ shows the systematic errors in the Kanizsa task. The subjects consistently 


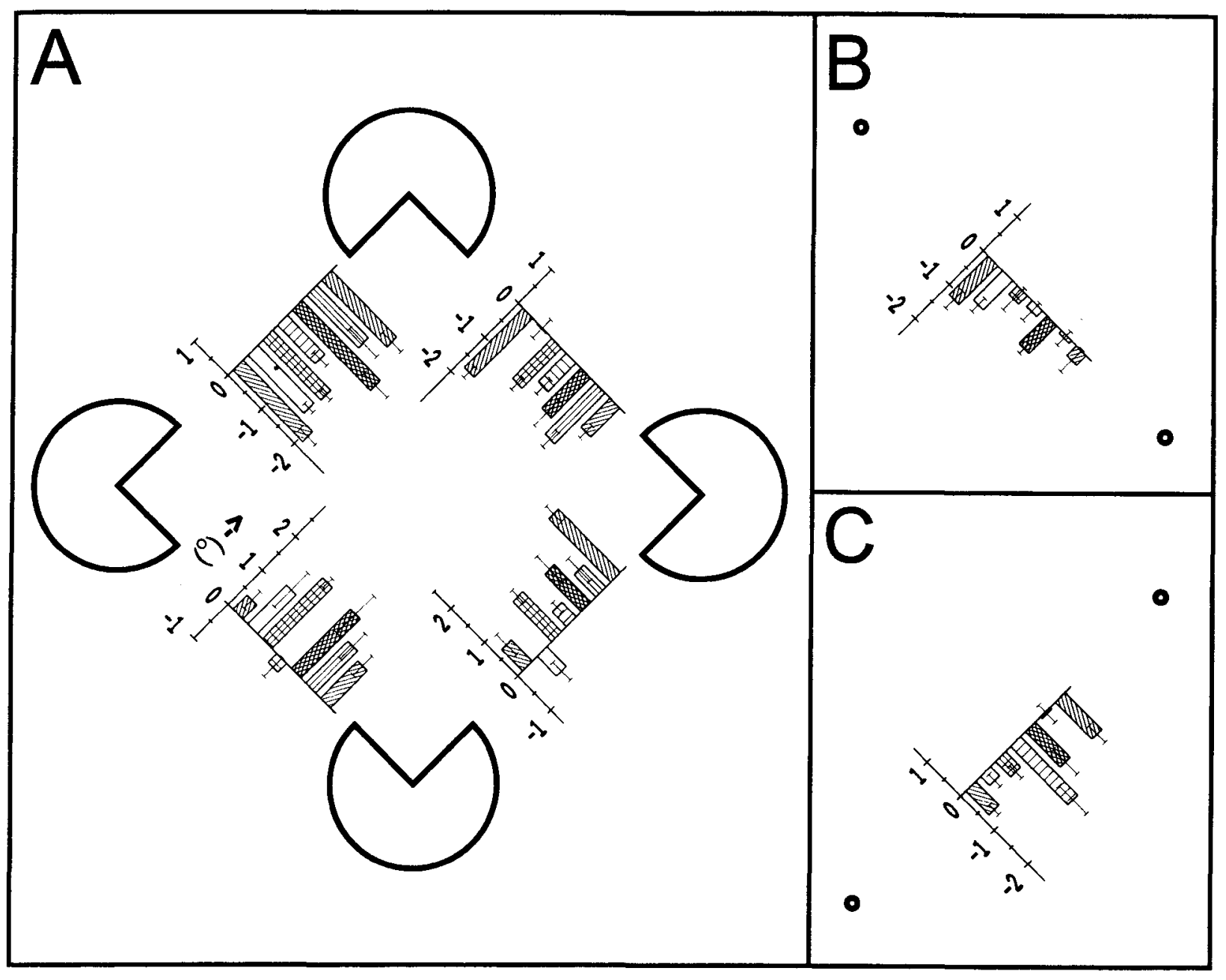

Figure 2. Results of Experiment 1 superimposed on sketches of the stimuli. In the experiment, white figures were shown on a circular black background, the pacmen had a radius of $2 \mathrm{~cm}\left(1.9^{\circ}\right.$ of visual angle), the distance between the centers of the pacmen or the two outer dots was $10 \mathrm{~cm}\left(9.5^{\circ}\right.$ of visual angle), the gap between two pacmen was $6 \mathrm{~cm}\left(5.7^{\circ}\right.$ of visual angle), and a dot was $0.27 \times 0.27$ $\mathrm{mm}^{2}\left(0.26^{\circ} \times 0.26^{\circ}\right.$ of visual angle $)$. Bars indicate mean systematic errors of 1 subject for the corresponding dot location. Panel A combines the results of the four stimuli where the adjustable dot appeared near one of the illusory lines of the Kanizsa diamond. Panel $B$ presents the oblique three-dot alignment task slanted to the left, and panel $\mathrm{C}$ the oblique three-dot alignment task slanted to the right. The systematic errors are expressed in degrees $\left(^{\circ}\right)$ of the angle between the straight line connecting the outer two markers and the straight line connecting the average end position of the dot and the center of one of the outer markers (see also Figure 5). Whiskers indicate standard errors of the mean. The averages and standard errors mean were based on 10 (L.V., P.E., M.V.), 11 (Y.M., R.B., A.S.), or 12 (N.S.) repeated measurements. Different subjects are denoted by different bar patterns: from left to right, Y.M. (lines slanted to the left), N.S. (clear), R.B. (squares), L.V. (horizontal lines), P.E. (diamonds), M.V. (vertical lines), and A.S. (lines slanted to the right).

misplaced the dot-that is, they did not place it on the contour but generally placed it too far toward the center of the Kanizsa diamond. The standard deviation is about $0.8^{\circ}$, on average, over edges and subjects.

Figures $2 \mathrm{~B}$ and $2 \mathrm{C}$ show the systematic errors in the three-dot alignment task. The subjects systematically placed the middle dot below the actual straight line that could connect the outer dots. These results are in agreement with the $0.5^{\circ}-1^{\circ}$ systematic errors in comparable experiments by Sittig and De Graaf (1994). The standard deviation is about $0.8^{\circ}$, on average, over edges and subjects.

The general impression is that the systematic errors in the Kanizsa diămond are larger than in the three-dot alignment task [comparing absolute size: paired two-tailed $t$ test, $n=28, t(27)=3.7, p<.01]$ and that they are directed toward the inner part of the Kanizsa diamond (sign test, $n=28, t=26, p<.001$ ). This means that in the lower half of the Kanizsa diamond, the errors in alignment are in the opposite direction to the errors in alignment in the oblique three-dot task. There appears to be no systematic difference in the standard deviations between these two tasks.

The half Kanizsa diamonds. While performing the task with a half Kanizsa diamond, about half of the subjects no longer reported seeing an illusory diamond. Subjects Y.M., M.V., and A.S. reported that they perceived a part of a diamond while performing the alignment task, subjects L.V. and P.E. reported that they were 


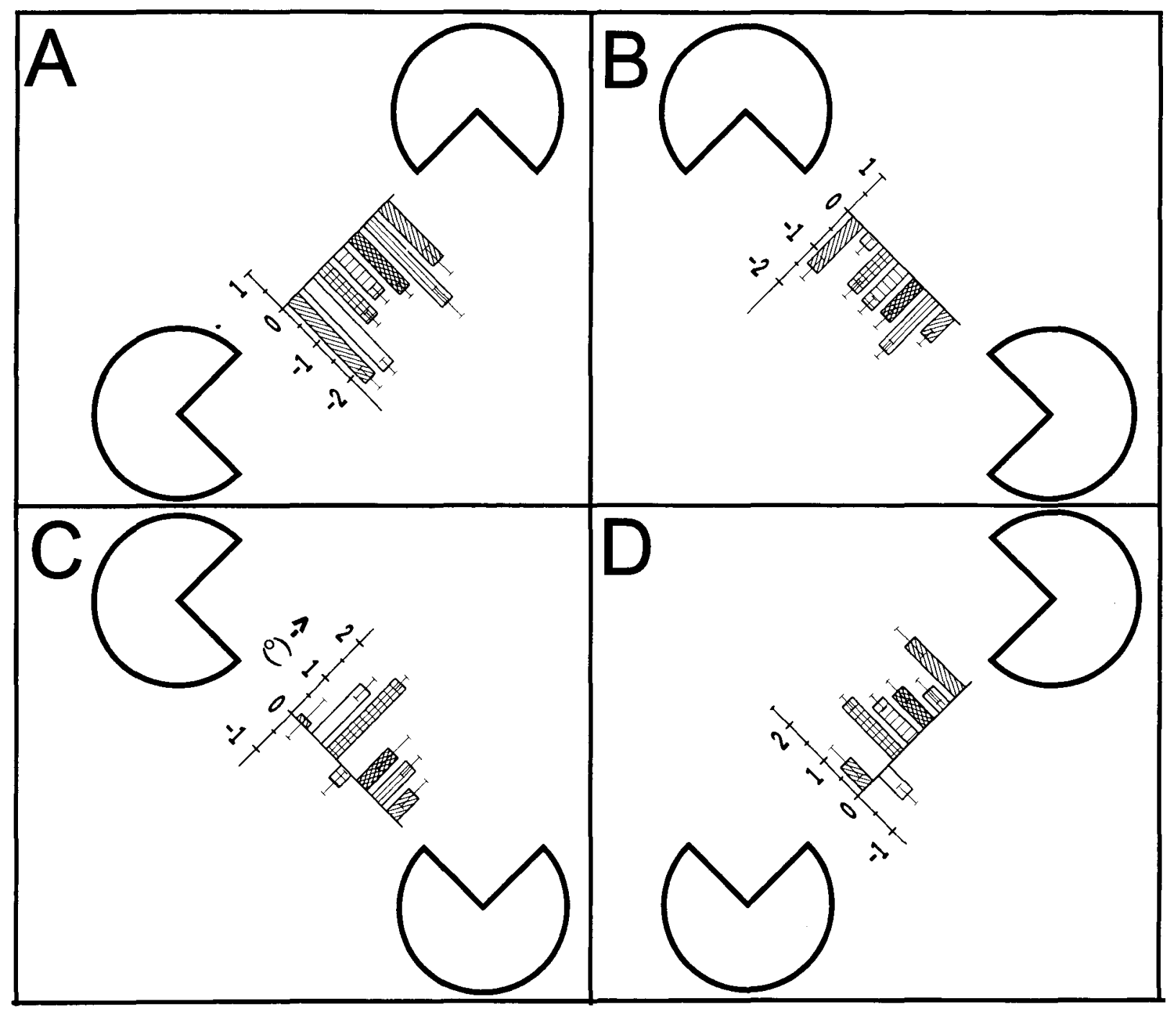

Figure 3. The results of Experiment 1. The data are presented as in Figure 2. Panel A presents the left top half of diamond, panel B the right top half of diamond, panel $C$ the left bottom half of diamond, and panel $D$ the right bottom half of diamond. Different subjects are denoted by different bar patterns: from left to right, Y.M. (lines slanted to the left), N.S. (clear), R.B. (squares), L.V. (horizontal lines), P.E. (diamonds), M.V. (vertical lines), and A.S. (lines slanted to the right).

able to see a part of a diamond surface if they concentrated on seeing it (which they usually did not when performing the alignment task), and subjects N.S. and R.B. reported that they only saw a vague dark-darker transition. Despite these differences between the subjects, there was no systematic difference in aligning the dot.

The systematic errors for all the subjects with the half Kanizsa diamonds are plotted in Figures 3A-3D. As one can see, the half-Kanizsa-diamond tasks led to an individual pattern of systematic errors that is very similar to the individual pattern observed in the Kanizsa-diamond task (2A). ${ }^{1}$ For example, the only subject with an average negative systematic error in the lower left contour in the Kanizsa-diamond task is again the only subject with an average negative systematic error in the lower left half-Kanizsa-diamond task. The systematic errors do not seem to depend on whether two or four inducing disks are shown to the subject. This is supported by the results of the analysis of variance (ANOVA) we performed. [The ANOVA showed that the hypotheses that all the subjects respond in the same way and that all the contours induce the same systematic errors could be rejected $(p<.05)$. The hypothesis that the half-Kanizsa-diamond task and the Kanizsa-diamond task are the same for corresponding contours and subjects could not be rejected $(p=.90)$.] Apparently, only the nearest two pacmen determine how a dot is aligned with an illusory contour.

The false Kanizsa diamonds. Figures $4 \mathrm{~A}$ and $4 \mathrm{~B}$ show the results plotted for a dot that had to be aligned to two pacmen in a false Kanizsa diamond. None of the subjects reported seeing a contour except subject Y.M., who claimed to see a vague line. The subjects generally put the dot below the straight line connecting the contour of two pacmen in these false Kanizsa diamonds. The 
Table 1

Summary of the Results of Experiment 1

\begin{tabular}{|c|c|c|c|c|}
\hline \multirow{2}{*}{$\begin{array}{l}\text { Stimulus and Location } \\
\text { of the Adjustable Dot }\end{array}$} & \multirow{2}{*}{$\begin{array}{l}\text { Mean Value of } \\
\text { Subject Means }\end{array}$} & \multirow[b]{2}{*}{$S D\left(^{\circ}\right)$} & \multicolumn{2}{|c|}{$\begin{array}{c}\mathrm{H}_{0}: \text { mean value }=\text { zero } \\
\text { Two-Tailed } t \text { Test }\end{array}$} \\
\hline & & & Value of $p$ & Value of $t(6)$ \\
\hline Kanizsa diamond: right top & -1.1 & 0.6 & $<.005$ & 5.0 \\
\hline Kanizsa diamond: right bottom & 0.8 & 0.8 & $<.05$ & 2.6 \\
\hline Kanizsa diamond: left top & -1.8 & 0.4 & $<.0001$ & 11.9 \\
\hline Kanizsa diamond: left bottom & 1.0 & 0.7 & $<.05$ & 3.7 \\
\hline Half Kanizsa diamond: right top & -1.0 & 0.4 & $<.001$ & 6.4 \\
\hline Half Kanizsa diamond: right bottom & 0.8 & 0.7 & $<.05$ & 2.7 \\
\hline Half Kanizsa diamond: left top & -1.8 & 0.5 & $<.0001$ & 10.0 \\
\hline Half Kanizsa diamond: left bottom & 0.8 & 0.8 & $<.05$ & 2.8 \\
\hline Three dot: slanted to the right & -0.8 & 0.6 & $<.05$ & 3.5 \\
\hline Three dot: slanted to the left & -0.5 & 0.4 & $<.05$ & 3.3 \\
\hline False Kanizsa diamond: right top & -0.8 & 1.0 & .07 & 2.2 \\
\hline False Kanizsa diamond: right bottom & -0.3 & 0.9 & .47 & 0.8 \\
\hline False Kanizsa diamond: left top & -0.7 & 0.4 & $<.01$ & 4.7 \\
\hline False Kanizsa diamond: left bottom & -0.3 & 0.5 & .12 & 1.8 \\
\hline
\end{tabular}

average systematic errors over all the subjects are not significantly different from zero for three of the four "edges" (see Table 1). The average size and direction are comparable with those in the three-dot alignment task. An individual pattern comparable with those observed with the Kanizsa diamond and the half Kanizsa diamonds is not apparent. The measured standard deviation is about $1^{\circ}$, on average, over all edges and subjects.

\section{Discussion}

The systematic errors measured in the Kanizsa-diamond task do not resemble those measured in the three-dot alignment task, although the standard deviations do not seem to differ. The results with the Kanizsa diamond and the half Kanizsa diamond do show a strong similarity, despite the fact that what subjects claimed to see could vary. Therefore, it would seem less likely that the system-

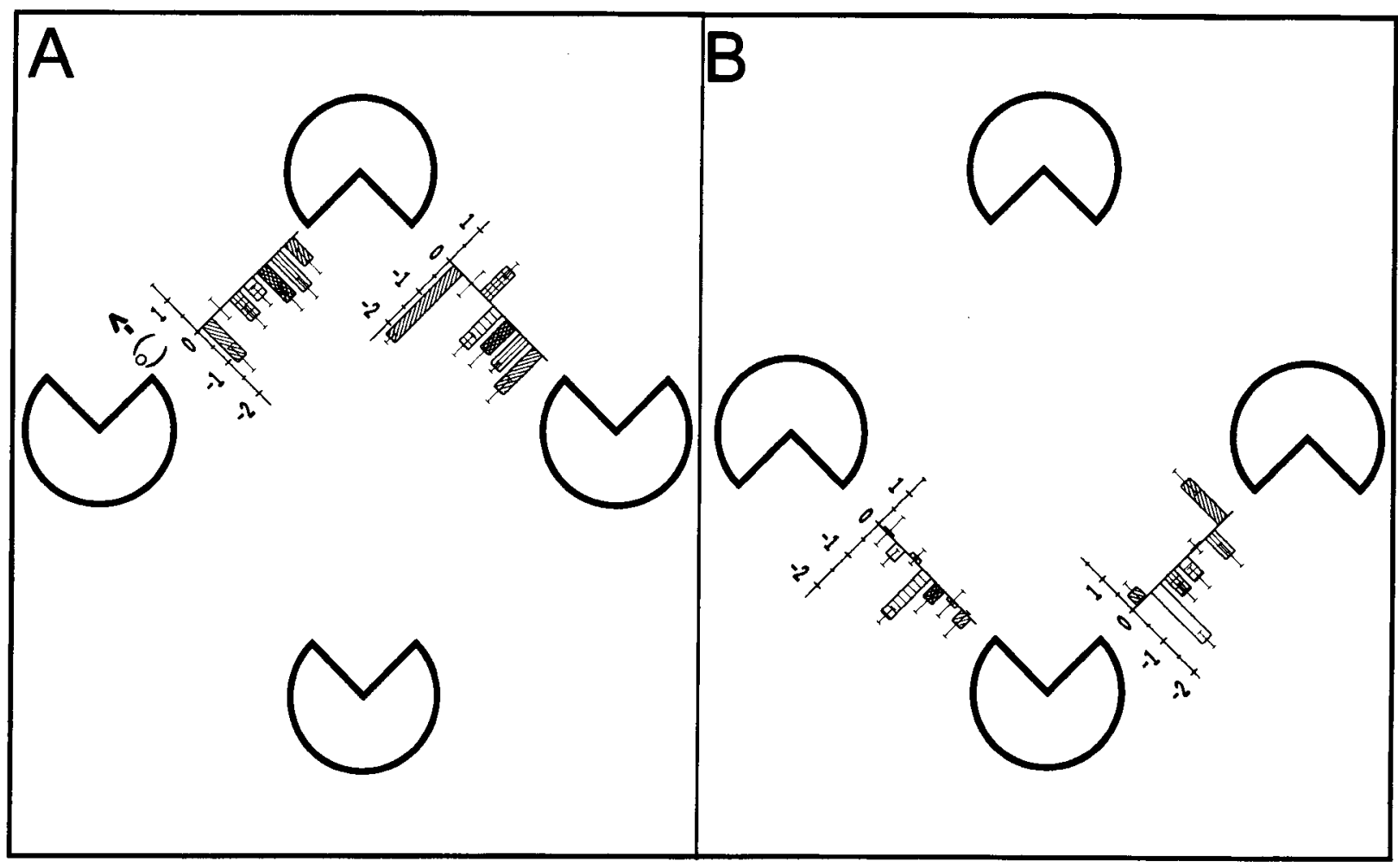

Figure 4. The results of Experiment 1. The data are presented as in Figure 2. Panel A combines the results of the two stimuli where the adjustable dot appeared near to midway between two pacmen in the top half of a false Kanizsa diamond. Panel $B$ combines the results of the two stimuli where the adjustable dot appeared near to midway between two pacmen in the bottom half of a false Kanizsa diamond. Different subjects are denoted by different bar patterns: from left to right, Y.M. (lines slanted to the left), N.S. (clear), R.B. (squares), L.V. (horizontal lines), P.E. (diamonds), M.V. (vertical lines), and A.S. (lines slanted to the right). 


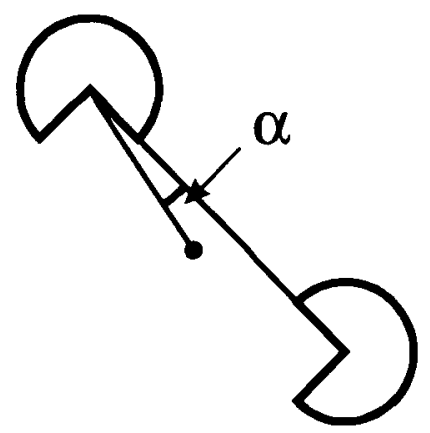

Figure 5. The angle of deviation, $\alpha$, is the angle between the straight line that connects the centers of two markers and the straight line that connects the dot with the center of one marker.

atic errors measured with the Kanizsa diamond are illusion dependent.

The rationale for including the false Kanizsa diamonds was as follows. Any systematic errors with the Kanizsa diamond could be induced by the illusion. The false Kanizsa diamond consists of the same markers as the Kanizsa diamond, but now no diamond can fit in the gaps. Therefore, no illusory surface should be perceived. If systematic errors with the Kanizsa diamond are illusion dependent, the false Kanizsa diamond should lead to a different pattern. This is what we observed. Nevertheless, we cannot yet conclude that a large part of the systematic errors with the Kanizsa diamond are illusion dependent. A single pacman may trigger mechanisms related to illusory form perception. This implies that the pacmen in the Kanizsa diamond and half Kanizsa diamond enhance each other's effect and in the false Kanizsa diamond compensate for each other's effect. This line of reasoning is consistent with that of Dresp and Bonnet (1993), who measured an increased light detection threshold near an illusory border and a slightly reduced light detection threshold right on the illusory border. They continued to find this effect when two pacmen were presented, or even only one.

\section{EXPERIMENT 2}

In the previous experiment, the data show that there was a Kanizsa-configuration-dependent bias with the Kanizsa diamond. With the three-dot alignment task, there was an orientation-dependent bias (Sittig \& de Graaf, 1994), but there could not have been a Kanizsa-configurationdependent one. Could the systematic errors in the Kanizsadiamond task be the result of the sum of two biasesthat is, the sum of an orientation bias (as occurs in the three-dot task) and a Kanizsa-configuration-dependent bias (induced by the pacmen, directed to the center)? This hypothesis would explain why, with the Kanizsa diamond, the systematic errors in the top half of the diamond are generally larger than those found in the lower half of the diamond. If the two effects add up, they would cooperate in the top half of the Kanizsa diamond but work against each other in the lower half. Such an additive ef- fect would also explain why, on average, the adjustable dot is placed slightly too low in the false Kanizsa diamond. If the pacmen compensate for each other's effect, only the orientation dependent bias remains. We know that the orientation-dependent bias disappears under a vertical or horizontal orientation (Sittig \& de Graaf, 1994). This means that when subjects are asked to align a dot to a contour of an illusory square, with the contours thus oriented vertically and horizontally, we should find systematic errors that are a result of the Kanizsa-configuration bias only. If the hypothesis of additive effects holds, these systematic errors are expected to be as large as the systematic errors found in the top half of the Kanizsa diamond minus the systematic errors in the oblique three-dot alignment task. We investigated this in Experiment 2.

\section{Method}

Five of the previous 7 subjects ( 2 female, 3 male) participated in this experiment. The experiment differed from Experiment 1 only in the stimuli. Now, all markers could be connected via horizontal or vertical straight lines, and we used only the Kanizsa square and two dots marking a horizontal or a vertical line (see Figure 6). All the stimuli were presented 10 (P.E.) or 12 (Y.M., N.S., R.B., M.V.) times. The convention for signs is that a positive value for the errors from a vertical-indicated "line" means that the dot was placed too far to the left, and a positive value for the errors from a horizontalindicated "line" again means that the dot was placed above the actual straight line.

\section{Results}

Most subjects volunteered the information that this experiment was easier to do than the previous one. The systematic errors and standard errors of the mean were plotted for all subjects and for each stimulus in Figure 6, as in Figure 2. Both the systematic errors (average size, $0.6^{\circ}$ ) and the variable errors (average standard deviation, 0.44 ) with the Kanizsa square were much smaller than those in Experiment 1. The systematic errors lie within the Kanizsa square in 16 of the 20 cases. Thus, a small but significant effect remains (one-sided sign test, $p<.01$ ). Table 2 lists the systematic errors averaged over subjects per indicated "line." We predicted that the size of the systematic errors would be the difference between the systematic errors with the Kanizsa diamond and the systematic errors with the three-dot alignment task, measured under the same orientation. For each of the subjects, we calculated the average predicted size of the systematic errors and compared these with the measured systematic errors with the Kanizsa square. The systematic errors with the Kanizsa square are smaller than those we predicted via our hypothesis (paired two-tailed $t$ test, $n=20, p<.001$ ). In the three-dot alignment task, the systematic errors were also almost zero, and the average standard deviation of $0.33^{\circ}$ is smaller than that in the oblique alignment task. These results are in agreement with those of Sittig and De Graaf (1994).

\section{Discussion}

The subjects did not place the dot as far toward the center of the Kanizsa square as they did with the Kanizsa 


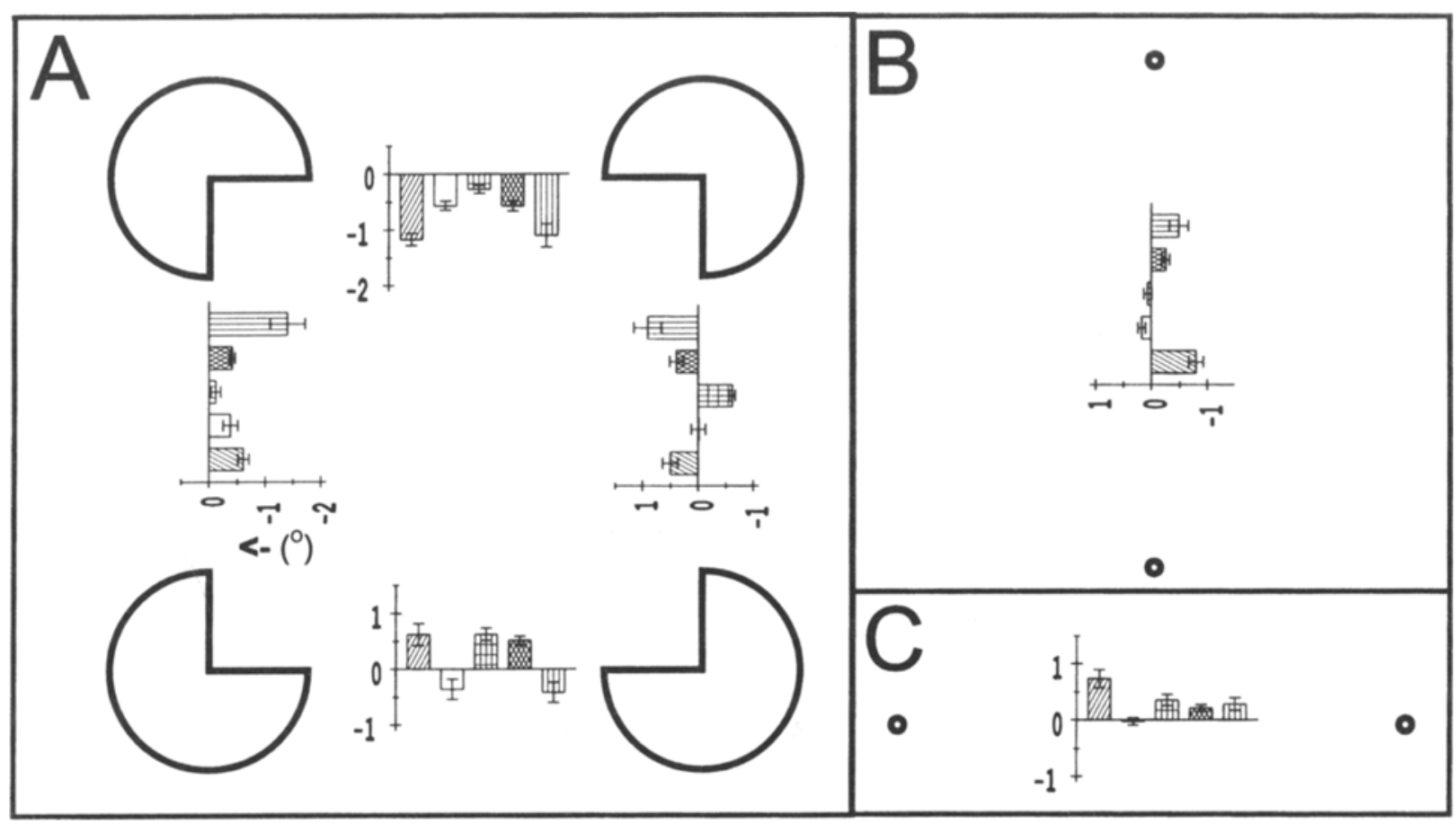

Figure 6. The results of Experiment 2. The data are presented as in Figure 2. In Panel A, the four stimuli in which an adjustable dot appeared near one of the illusory contours of the Kanizsa square are combined. Panel B presents the vertical three-dot alignment task, and panel $\mathrm{C}$ the horizontal three-dot alignment task. The systematic errors are indicated in degrees $\left(^{\circ}\right)$ of the angle between the straight line connecting the outer two markers and the straight line connecting the average position of a dot, placed by the subject, and the center of one of the outer markers (see also Figure 5). Whiskers indicate standard errors of the mean. The averages and standard errors mean were based on 10 (P.E.) or 12 (Y.M., N.S., R.B., M.V.) repeated measurements. Different subjects are denoted by different bar patterns: from left to right, Y.M. (lines slanted to the left), N.S. (clear), R.B. (squares), L.V. (horizontal lines), P.E. (diamonds), and M.V. (vertical lines).

diamond. Thus, the systematic (and variable) errors found in the Kanizsa-diamond task appear to be subject to an oblique effect as well. The systematic errors are even smaller than would be expected by assuming that the results with the Kanizsa diamond were the summed effects of two independent biases: a Kanizsa-configurationdependent bias and an orientation-dependent bias. Thus, the two biases are not combined in a simple additive way.

A remaining bias to place the dot too far toward the center of the square is consistent with the results reported by Pomerantz et al. (1981). They presented a Kanizsa square to their subjects and asked them whether an appearing dot was inside or outside the square. They measured reaction times and the percentages of correct responses. Although in their case, the dot appeared at distances twice as large as the average systematic error made by our subjects, Pomerantz et al. did report that their subjects more often wrongly claimed the dot to be outside the square than the other way around. They did not offer an explanation for this finding.

The results of this second experiment can exclude three other possible explanations for the systematic errors in aligning a dot to an oblique illusory contour. The first could be that the perceived surface is smaller than the square that fits within the pacmen. The light-dark border of the pacmen could have been perceived to be fur- ther away from the white pacmen than the actual border. Second, the square could be perceived as smaller than the square that can be fitted into the pacmen, because it is perceived to be closer than the pacmen (Coren, Porac, \& Theodor, 1986). This is consistent with the explanation of the Kanizsa illusion by means of the figure-ground segregation (Petry \& Meyer, 1987). These two suggestions, however, do not predict the strong orientation dependency we observed.

A third possible explanation concerns the role of binocular viewing. The stimuli were always viewed binocularly in our experiments. When viewing the contour of a surface with both eyes, one is able to look beyond the contour with one of the eyes. If the dot is perceived to be at a different depth from that of the illusory surface, it can

Table 2

Summary of the Results of Experiment 2

\begin{tabular}{lcc}
\hline $\begin{array}{l}\text { Stimulus and Location } \\
\text { of the Adjustable Dot }\end{array}$ & $\begin{array}{c}\text { Mean Value of } \\
\text { Subject Means }\end{array}$ & $S D\left(^{\circ}\right)$ \\
\hline Kanizsa square: top & -0.7 & 0.4 \\
Kanizsa square: left & -0.6 & 0.5 \\
Kanizsa square: right & 0.2 & 0.6 \\
Kanizsa square: bottom & 0.2 & 0.5 \\
Three dots: horizontal & 0.3 & 0.3 \\
Three dots: vertical & -0.3 & 0.4 \\
\hline
\end{tabular}


lead to a bias in perceived frontoparallel position. Since one eye is displaced horizontally with respect to the other, it would predict maximal systematic errors for an illusory contour under vertical orientation and minimal systematic errors for an illusory contour under horizontal orientation. Contrary to this, we observed the largest systematic errors under oblique orientation. Therefore, binocular viewing also fails to explain the observed effect.

\section{EXPERIMENT 3}

One of the results of Experiment 1 was to show that the systematic errors were the same for all the subjects, irrespective of whether two or four pacmen were shown. Note that this finding does not depend on what the subjects saw when they were presented with the half Kanizsa diamonds. We concluded that only the two nearest pacmen determine the systematic errors. Perhaps the shape of the individual pacmen causes systematic errors. In the next experiment, we examined the influence of a single pacman.

\section{Method}

The same 5 subjects as those from Experiment 2 participated in this experiment. It was similar to Experiments 1 and 2 in that the subjects were asked to align a dot, but now only one pacman at a time was shown. The contours of the gap of the single pacmanshaped markers were oriented obliquely, as in Experiment 1. The subjects were instructed to place the dot on the straight line that would extend from the nearer of the two straight contours of the presented pacman. They had to extrapolate $4 * 2=8$ contours of pacmen: both oblique contours of each single pacman that, when presented together, would form a Kanizsa diamond. The distance between the dot and the pacman and the dimensions of the pacmen were the same as those in the previous experiments. The single pacmen appeared in the same position as in the Kanizsa diamond. Within any one set, the eight possible extrapolations were presented in quasi-random order. A set of stimuli was presented 10 (P.E.) or 12 (Y.M., N.S., R.B., M.V.) times.

\section{Results}

In Figure 7 , for each subject and each marker, the average systematic errors were plotted on the line extending the contour of a pacman. This is similar to Figure 2. As can be seen in Figure 7, with one pacman the subjects

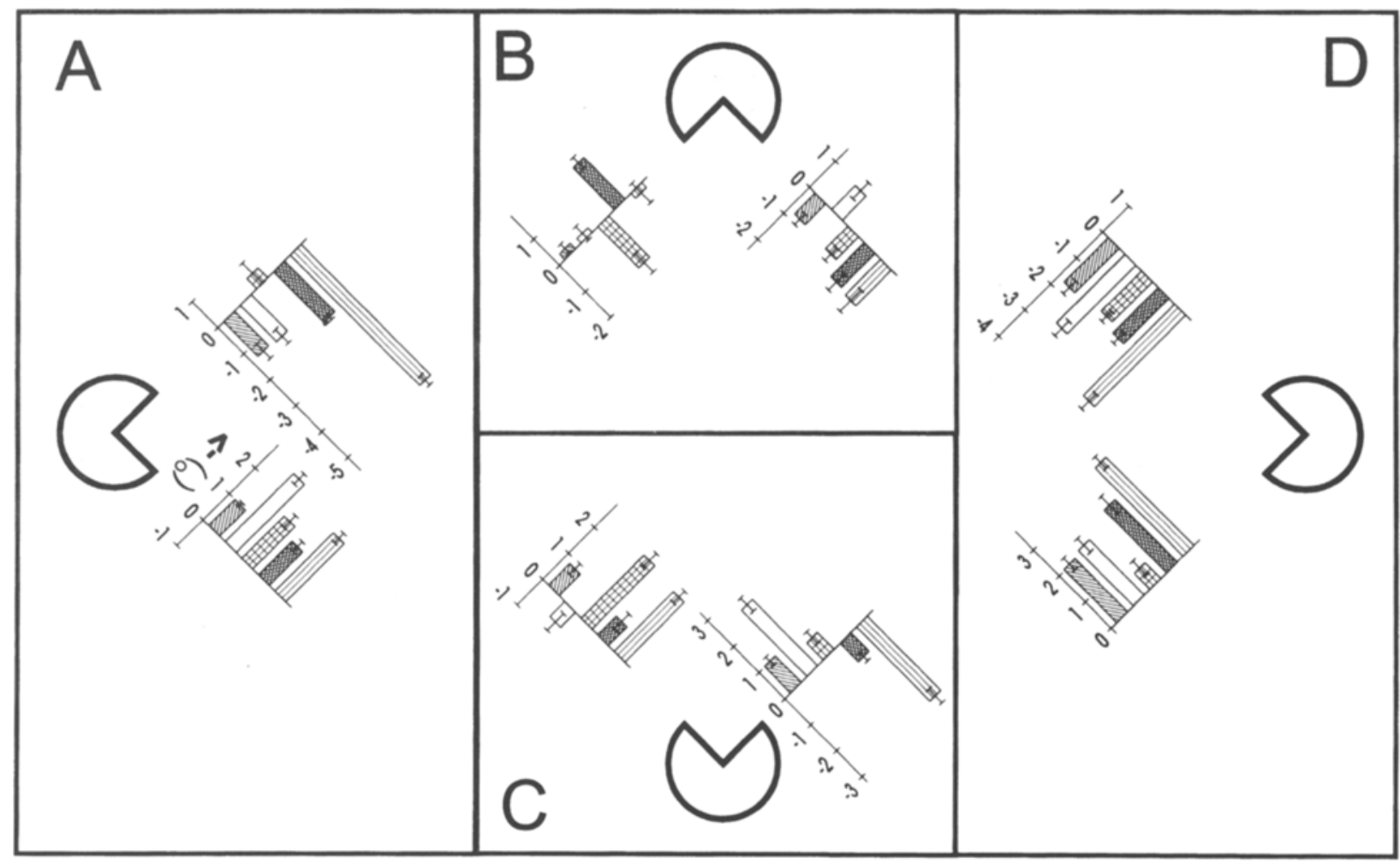

Figure 7. The results of Experiment 3. The results are presented in almost the same way as in Figure 2. Bars indicating average systematic errors are plotted for all the subjects and are superimposed on the straight line that could extend the straight contour of a pacman. Panel A shows the combined results of extrapolating a contour toward the upper right and of extrapolating a contour toward the lower right of the left single pacman. Panel B shows the combined results of extrapolating a contour toward the lower right and of extrapolating a contour toward the lower left of the top single pacman. Panel $C$ shows the combined results of extrapolating a contour toward the upper right and of extrapolating a contour toward the upper left of the bottom single pacman. Panel D shows the combined results of extrapolating a contour toward the upper left and of extrapolating a contour toward the lower left of the right single pacman. The systematic errors are indicated in degrees $\left({ }^{\circ}\right)$ of the angle between the straight line extending the contour of a pacman and the straight line connecting the average end position of the dot and the center of the pacman. Whiskers indicate standard errors of the mean. The averages and standard errors of the mean were based on 10 (P.E.) or 12 (Y.M., N.S., R.B., M.V.) repeated measurements. Different subjects are denoted by different bar patterns: from left to right, Y.M. (lines slanted to the left), N.S. (clear), R.B. (squares), P.E. (diamonds), and M.V. (vertical lines). 
also put the dot too far toward the inner part of the diamond. The systematic errors vary more among subjects and contours than do those in Experiment 1 for the same 5 subjects: The average systematic errors vary from $1.6^{\circ}$ outside the diamond to $5.0^{\circ}$ inside the diamond in the single pacman experiment, as compared with $0.7^{\circ}$ outside the diamond and $2.4^{\circ}$ inside the diamond with the half Kanizsa diamonds in Experiment 1. Thus, the systematic errors from the straight extrapolation of the contour of one single pacman are comparable with those found with the half Kanizsa diamonds of Experiment 1 but are somewhat more variable. The average measured standard deviation equals $0.8^{\circ}$ and is similar to that observed in Experiment 1.

Are the systematic errors found with the half Kanizsa diamonds equal to the average effect of extrapolating the two single pacmen that form this half diamond? In Figure 8 we plotted, for each contour and each subject, the systematic errors with the half Kanizsa diamonds against the corresponding average systematic errors with the two single pacmen that together form that half Kanizsa diamond. The line of perfect correspondence is drawn as a solid line. The broken line indicates the best fit through the plotted points. This best fit has a slope equal to $1.1 \pm$ 0.2 and intersects the $y$-axis at $0.4^{\circ} \pm 0.2^{\circ}$. Thus, the line of perfect correspondence lies within the confidence limits of the fit. In the same way, the systematic errors found when extrapolating a contour of a single pacman can also explain the systematic errors that were found with the false Kanizsa diamond in Experiment 1.

\section{Discussion}

From this last result, we can conclude that the observed systematic errors in aligning a dot with a contour of a Kanizsa diamond can be explained as the average systematic error in extrapolating the two pacmen that indicate that contour. Why would our subjects extrapolate when asked to put a dot on an illusory contour? This could indicate that perception of the illusory surface and the location of the dot cannot be done simultaneously. This hypothesis is supported by two facts known from the literature. First, the dot itself might also function as an inducing element. In the Kanizsa square, the pacmen induce the perception of a square. Single dots cannot evoke an illusory surface by themselves (Gregory, 1972). However, when dots are shown in combination with line elements or pacmen, they do influence the contour (Gregory, 1972; Kanizsa, 1976). Although our subjects never complained that the task was impossible, it might be difficult to judge the position of a dot relative to an illusory

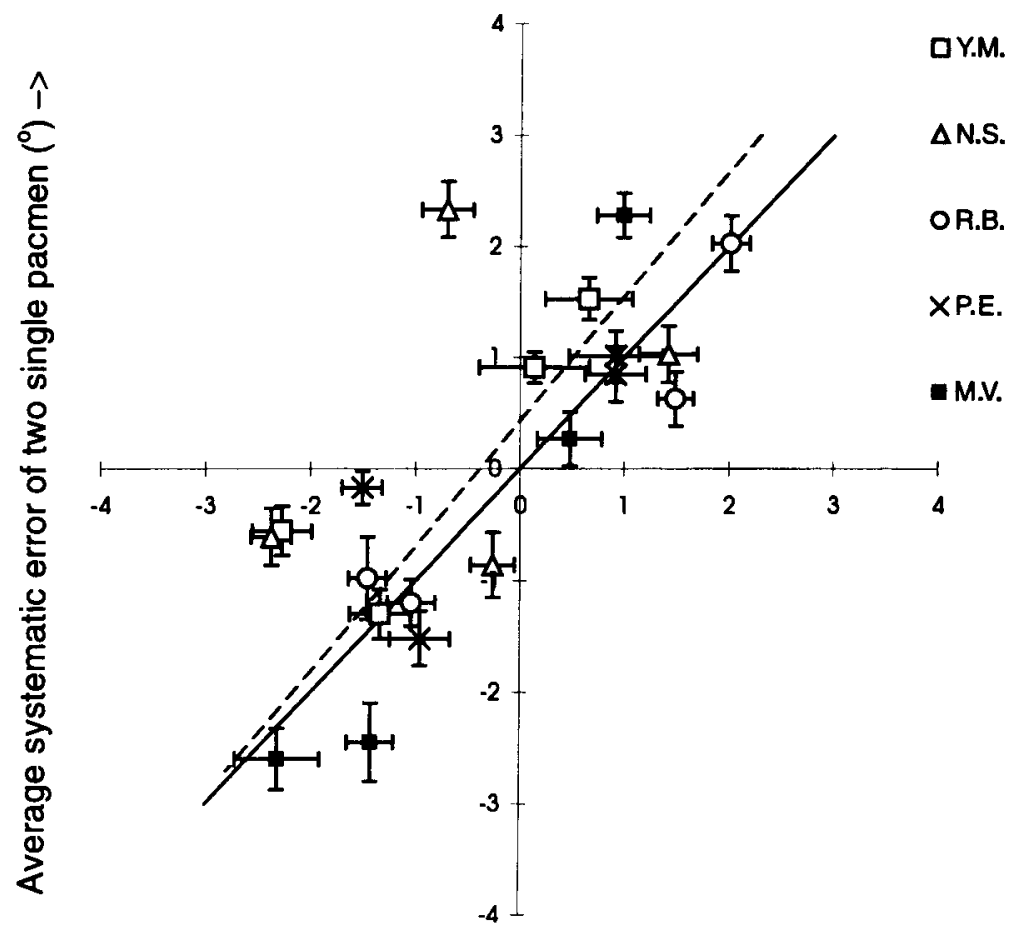

Systematic error half Kanizsa diamond $\left({ }^{\circ}\right) \rightarrow$

Figure 8. The systematic errors with the half Kanizsa diamonds for each contour and each subject were plotted against the corresponding average of systematic errors with two single pacmen that together form that half Kanizsa diamond. The straight line $\{y=x\}$ indicates the line of perfect correspondence. The dashed line $[y=(1.1 \pm 0.2) x+(0.4 \pm 0.2)]$ indicates the best fit through the plotted points. The whiskers indicate the standard errors. 
contour when that same dot codetermines that illusory contour. This problem would lead to larger variable errors in our experiment, since the dot appeared randomly inside and outside the illusory surface. Second, Kanizsa already noticed that the illusory contours fade when a person concentrates on looking at one of the illusory contours and that the illusory contours are seen most clearly when the configuration is "watched as a whole." In our experiments, the task involved only one of the contours at a time, drawing attention to that one.

\section{EXPERIMENT 4}

The results of the previous experiment suggest that the subjects did not perform the task in Experiment 1 as they were instructed - that is, to align the adjustable dot with the illusory contour. This raises the question of the role, if any, of the illusory contour in the performance of that task. There may be no influence of the illusion, perhaps because it is not seen when the dot is being aligned. Why, then, is there such a difference in results between the task with the dots and the one with the pacmen? This could imply an influence of the (asymmetry of the) shape of objects from which the position has to be judged. Alternatively, the mechanisms for figure-ground segregation are triggered already by one single pacman, as Dresp and Bonnet (1993) suggest. To test this, we presented the subjects with diamonds, which were indicated by pacmen, Xs, Ls, or dots, and asked them to place a dot on the average extrapolation of the edges of the pacmen, Xs, or Ls or to align the dot with the two other dots that were nearest. It is known that the Xs and Ls do not evoke the perception of an illusory surface (Kanizsa, 1979) but do differ in (a-) symmetry. The diamond indicated by the dots was included to bridge the gap between the threedot alignment task and the Kanizsa-diamond task.

\section{Method}

This experiment was set up in a way that was similar to that of the previous experiments. Five subjects participated, 4 who had participated in the previous experiments and 1 new naive subject (K.A.). All the subjects gave informed consent. The subjects were told that they would be presented with diamond-like shapes indicated by four Xs, four Ls, four pacmen, or four dots. They were instructed to place the dot on the intersection of the apparent straight lines that would extend from the nearest two straight edges or, in the case of the dots, to place the dot on the straight line that would connect the nearest two dots. Each stimulus was presented 10 times and appeared at a location in the center of the screen, with some random jitter in that location from trial to trial.

\section{Results}

Figure 9 shows the results for a diamond indicated by four Xs or four Ls. The systematic differences from a straight line were small in both cases (about $0.4^{\circ}$ ), about the size of the standard error of the mean $\left(0.6^{\circ}\right.$, on average). However, there is a difference. With the Ls, the systematic errors were generally directed toward the center of the figure (sign test, $n=20, T=14, p<.005$ ), whereas with the Xs, the systematic errors seem to have been randomly distributed in either direction $[t$ test, $t(199)=1.62$, $p=.11]$.

Figure 10 shows the results for the dots. Surprisingly, the systematic errors were not always directed below the

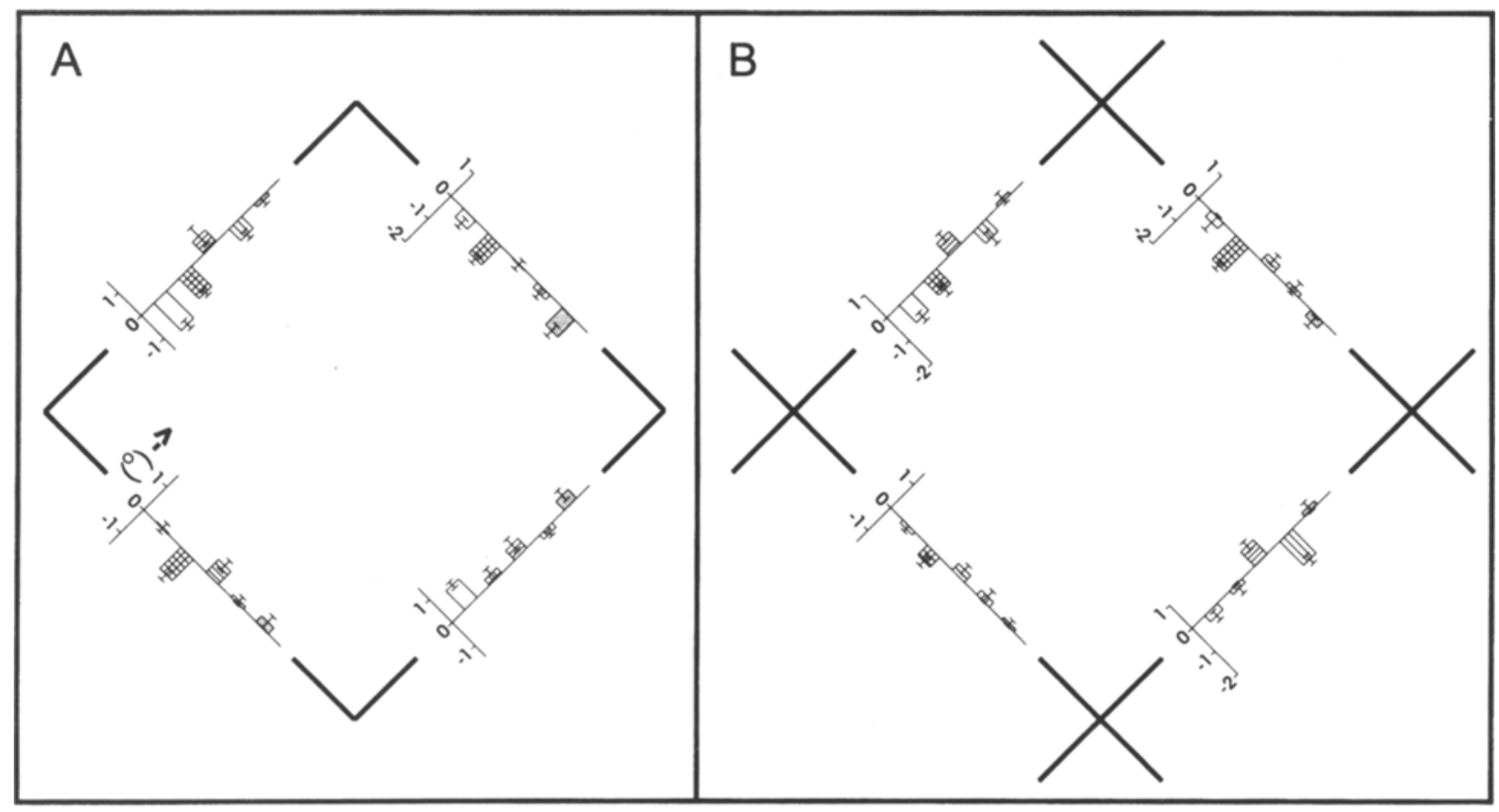

Figure 9. The results of extrapolating the two nearest edges of $L$-shapes (A) or $X$-shapes (B). The results are presented in the same way as those in Figure 2. Different subjects are denoted by different bar patterns: from left to right, N.S. (clear), R.B. (squares), L.V. (horizontal lines), M.V. (vertical lines), and K.A. (rightmost). 


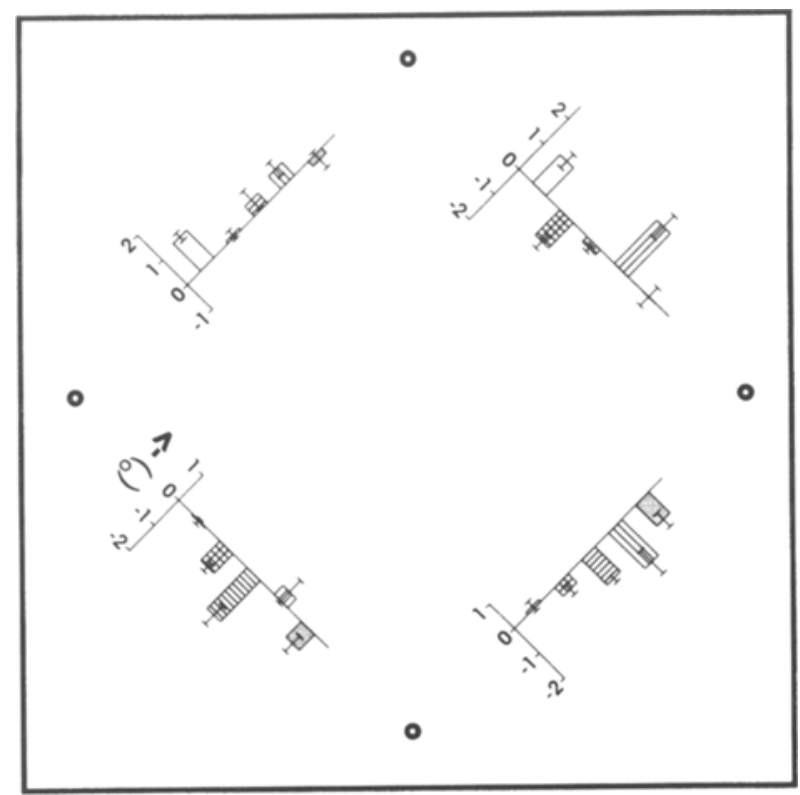

Figure 10. The results of aligning a dot with the two other dots closest to the adjustable one. Besides the adjustable dot, four other dots were visible, indicating a diamond. Different subjects are denoted by different bar patterns: from left to right, N.S. (clear), R.B. (squares), L.V. (horizontal lines), M.V. (vertical lines), and K.A. (rightmost).

straight line that could connect two dots [ $t$ test, $t(199)=$ $1.60, p=.11]$. This holds particularly for the systematic errors in the upper half of the diamond. The systematic errors generally seemed to be directed outside the figure $[t$ test, $t(199)=6.34, p<.001]$ and were about equal in size to those with the Ls and Xs. The standard deviations equaled $0.8^{\circ}$, on average, which is larger than those observed with the Ls and Xs.

The results with the pacman reproduce the results of Experiment 1; for all 5 subjects and all four edges of the diamond, the systematic errors were directed toward the center of the figure, and the systematic errors $\left(1.9^{\circ}\right.$ on average) were usually larger than the standard deviations $\left(0.9^{\circ}\right.$ on average). This reproduction of results occurs despite the fact that we now asked our subjects to extrapolate the two nearest edges of the pacmen. This is in agreement with the results from the single pacmen.

\section{Discussion}

Since large (and very significant) effects occur only with the pacman, we must assume that there is something special about it that causes these effects. One way in which pacmen differ from the other objects is that they can induce an illusory figure. However, this is not the only difference. The size of the luminant surface is different, and they have an incomplete circular edge. Nevertheless, the results do seem to suggest some influence of figureground segregation. Such an influence could also explain why the results with the dots are different when four, rather than two, fixed'dots are shown. However, this is only spec- ulation, because the deviations are small and Sittig and De Graaf (1994) found less clear effects when the dots could mark a straight oblique line downward, as compared with the same line's running upward.

The results of this experiment clearly demonstrate that relative localization depends on the shape of objects in relation to the location that has to be judged.

\section{GENERAL DISCUSSION}

In the introduction, we stated that there are many situations in which the human visual system can make judgments about intermediate positions. When judging relative location, the human visual system can judge whether three objects are aligned or are indicating a plane. In some cases, those objects can induce illusory contours (Schumann, 1900) - that is, contours that are perceived as if they were real. We wanted to know whether the visual system uses the same mechanisms for estimating the simplest connection between two objects irrespective of whether those objects are dots or pacmen. To attempt to answer this question, we compared the results of a psychophysical experiment in which a dot had to be aligned either to two markers that could be connected by a straight oblique line (two dots) or to an oblique illusory contour.

Prior to the experiment, we had anticipated two possible outcomes: The results of the two tasks would be very similar, indicating that the same mechanisms are used, or smaller systematic errors would occur in aligning a dot to an illusory contour, indicating the use of additional mechanisms that use the edges of the pacmen. The results of the two tasks were clearly different. In the oblique three-dot alignment task, the middle dot was systematically placed too low. This reproduces the Sittig and De Graaf (1994) results. In the alignment task with the illusory contour, rather larger systematic errors were made that were directed to the center of the illusory surface. From this we concluded that an additional mechanism is used that introduces its own bias.

There are a number of computational models that assume that illusory contours result from the same mechanisms that account for the perception of real form (Grossberg, 1994; Heitger \& von der Heydt, 1993; Marr, 1982). These models are supported by experimental results in which an illusory contour has a very similar effect to a real contour. Vogels and Orban (1987) have demonstrated the largest just-noticeable differences in orientation with oblique orientation for both real and illusory contours. Gregory (1972) demonstrated that the Poggendorff illusion also occurs when the parallel vertical lines are illusory. Smith and Over (1977) demonstrated that illusory contours, like real ones, can produce tilt aftereffects. If illusory and real contours are functionally equivalent at some level, we might have expected our subjects to be able to minimize the distance between the dot and the illusory contour, as is possible with a real contour. The mechanisms underlying the illusory contour could then have helped in localizing the dot. However, we did not 
observe a reduction in either the systematic errors or the variable errors when the Kanizsa diamond was perceived. Thus, the illusory contour does not help in localizing objects, and thus, in this case, illusory contours differ from real contours.

We then hypothesized that the Kanizsa configuration introduced its own bias and that this bias was linearly added to the one associated with the mechanisms related to relative localization. We rejected this hypothesis on the basis of Experiment 2, which demonstrated too strong an oblique effect in the results.

Experiment 1 also showed that the results of aligning a dot to an illusory contour did not differ whether four or only two pacmen were presented, whereas the strength of the illusion did differ. This raised questions about the role of a single pacman and of the illusory contour. Experiment 3 showed that the errors in aligning a dot to an oblique illusory contour can be described as the average result of interpolating the two nearest edges of the pacmen. This suggested that the difference in results between the pacmen and the dots was not caused by the illusion, but by the difference in symmetry of their shape. We tested this idea in our last experiment, where we asked subjects to extrapolate from pacmen, Ls, and Xs. The results with the pacmen were very similar to those in Experiment 1, and they were the only stimuli with clear large effects. The Ls did show a much weaker effect, also directed toward the center of the figure, whereas no systematic or significant effect occurred with the Xs. This suggests that there is something special about the pacman shape that causes large effects. The pacmen have a larger bright surface and an incomplete circular edge, which the other objects do not have. The pacmen are also the only objects which can induce an illusory surface by themselves. The question remains whether the bright surface and the incomplete circular edge of the pacmen cause large effects because they can induce illusory contours at the location of the perceived interpolation. One thing is clear: The shape of the markers influences the localization of objects relative to these objects. The influence of the shape of an object on a more global localization has been demonstrated (Brenner \& Smeets, 1995).

Our experiments were psychophysical experiments, and they can provide an explanation only at that level. There are also several reports concerning illusory contours of neural responses at a physiological level. However, to combine the results of these different levels of explanation is not a trivial task (Spillman \& Dresp, 1995), for several assumptions are needed. There are cells that are sensitive for T-junctions. These cells are active when there are pacmen in its visual field and not active when there are only dots in its visual field. There are cell recordings that show firing patterns sensitive to figure-ground segregation (Baumann, van der Zwan, \& Petherhans, 1997). We would expect the figure to be detectable from the ground at some neural level, because the direction of the systematic errors is always in the direction of the cen- ter of the figure, independent of orientation. Heitger and von der Heydt (1993) have made a neural model based on the firing patterns of complex cells. It would be interesting to measure the cell activity as a result of the same stimuli that we used. We predict that cells with a firing rate that is dependent on the position of the dot will show a change in firing pattern when the dot is just inside the figure and that the precise position of the dot when this happens is dependent on the orientation for which the cells are sensitive. The neural model of Heitger and von der Heydt strongly depends on the role of so called end-stop cells. One major difference between the pacmen and the Ls or Xs is that the pacmen also trigger end-stop cells because of the noncontinuation of the circular edge that is present in the pacmen. Perhaps this is reflected in the results of Experiment 4.

In conclusion, the experiments described in this paper indicate that there is a complex interaction between the shape of objects and the judgment of relative location. An illusory contour does not act in the same way as a real contour when a dot is aligned to an illusory contour. In this task, our subjects appeared to be interpolating the edges of the two nearest pacmen. The results clearly demonstrated that the shape of objects influences the extrapolation and interpolation (of the edges) of those objects.

\section{REFERENCES}

APPELLE, S. (1972). Perception and discrimination as a function of the stimulus orientation. Psychological Bulletin, 78, 266-278.

Baumann, R., van DER ZWAN, R., \& Petherhans, E. (1997). Figureground segregation at contours: A neural mechanism in the visual cortex of the alert monkey. European Journal of Neuroscience, 9 , $1290-1303$.

Brenner, E., \& Smeets, J. B. J. (1995). Moving one's finger to a visually specified position: Target orientation influences the finger's path. Experimental Brain Research, 105, 318-320.

Coren, S., Porac, C., \& Theodor, L. H. (1986). The effects of perceptual set on the shape and apparent depth of subjective contours. Perception \& Psychophysics, 39, 327-333.

DRESP, B., \& BonNET, C. (1993). Psychophysical measures of illusory form perception: Further evidence for local mechanisms. Vision Research, 33, 759-766.

GrEGORY, R. L. (1972). Cognitive contours. Nature, 238, 51-52.

GrossBerg, S. (1994). 3-D vision and figure-ground separation by visual cortex. Perception \& Psychophysics, 55, 48-121.

HeITGER, F., \& voN DER HEYdT, R. (1993). A computational model of neural contour processing: Figure-ground segregation and illusory contours. Proceedings of the 4th International Conference on Computer Vision (pp. 32-40). Washington, DC: IEEE Computer Society.

KaNizSA, G. (1955). Margini quasi-percetttivi in campi con stimolatzione omogena. Revista di Psicologia, 49, 7-30. [Translated in Petry \& Meyer, 1987]

KANIZSA, G. (1976). Subjective contours. Scientific American, 234, 48-52. Kanizsa, G. (1979). Organization in vision: Essays on Gestalt perception. New York: Praeger.

MARR, D. (1982). Vision: A computational investigation into the human representation and processing of visual information. New York: Freeman.

Petry, S., \& Meyer, G. E. (EDS.) (1987). The perception of illusory contours. New York: Springer-Verlag.

Pomerantz, J. R., Goldberg, D. M., Golder, P. S., \& Tetewsky, S. (1981). Subjective contours can facilitate performance in a reactiontime task. Perception \& Psychophysics, 29, 605-611. 
Schumann, F. (1900). Einige beobachtungen über zusammenfassung von gesichtseindrücken zu einheiten. Zeitung für Psychologie, 23, 132. [Translated in Petry \& Meyer, 1987]

SitTig, A. C., \& de GraAF, J. B. (1994). Orientation dependent misalignments in a visual alignment task. Vision Research, 34, 2195-2203.

SMITH, A. T., \& OVER, R. (1977). Orientation masking and the tilt illusion with subjective contours. Perception, 6, 441-447.

Spillman, L., \& Dresp, B. (1995). Phenomena of illusory form: Can we bridge the gap between levels of explanation? Perception, 24, 13331364.
Vogels, R., \& ORBan, G. A. (1987). Illusory contour orientation discrimination. Vision Research, 27, 453-467.

\section{NOTE}

1. The pacmen that indicated the half Kanizsa diamonds were at the same position on the screen as they were in the Kanizsa diamond. A pilot experiment ( 1 subject, the first author), however, showed that the systematic errors did not depend on the position of the half Kanizsa diamond on the computer screen.

(Manuscript received March 10, 1998;

revision accepted for publication September 21, 1999.) 